\title{
MONOPOLE CONDENSATES IN SEIBERG-WITTEN THEORY
}

\author{
Cihan Saçlıoğlu ${ }^{1,2}$ \\ ${ }^{1}$ Physics Department, Bog̃aziçi University \\ 80815 Bebek-İstanbul, Turkey \\ and \\ ${ }^{2}$ Feza Gürsey Institute \\ TUBITAK-Bog̃aziçi University \\ 81220 Çengelköy, Istanbul- Turkey
}

\begin{abstract}
A product of two Riemann surfaces of genuses $p_{1}$ and $p_{2}$ solves the Seiberg-Witten monopole equations for a constant Weyl spinor that represents a monopole condensate. Self-dual electromagnetic fields require $p_{1}=p_{2}=p$ and provide a solution of the euclidean Einstein-MaxwellDirac equations with $p-1$ magnetic vortices in one surface and the same number of electric vortices in the other. The monopole condensate plays the role of cosmological constant. The virtual dimension of the moduli space is zero, showing that for given $p_{1}$ and $p_{2}$, the solutions are unique.
\end{abstract}


In this note we present solutions of the Seiberg-Witten monopole equations $(\mathrm{SWME})$ [1]

$$
\begin{aligned}
& \not D_{A} \psi \equiv \gamma^{a} E_{a}^{\mu}\left(\partial_{\mu}+i A_{\mu}+\frac{1}{8} \omega_{\mu}^{b c}\left[\gamma_{b}, \gamma_{c]}\right) \psi=0,\right. \\
& F_{\mu \nu}^{+} \equiv \frac{1}{2}\left(F_{\mu \nu}+\frac{1}{2} \epsilon_{\mu \nu \alpha \beta} F^{\mu \nu}\right)=-\frac{i}{4} \psi^{\dagger}\left[\gamma_{\mu}, \gamma_{\nu}\right] \psi
\end{aligned}
$$

for which the 4-manifold $\mathcal{M}_{4}$ has the form $\Sigma_{p_{1}} \times \Sigma_{p_{2}}$ with $p_{1}+p_{2} \geq 2$, excluding $p_{1}=p_{2}=1$. The Weyl spinor $\psi$, which represents massless monopoles in the SWME, consists here of a single constant component $\psi_{1}\left(\psi_{2}\right)$, giving rise to a monopole (antimonopole) condensate. Physically, $p_{1}-1\left(p_{2}-1\right)$ is the number of magnetic (electric) vortices in $\Sigma_{p_{1}}\left(\Sigma_{p_{2}}\right)$. Remarkably, the most symmetric case with self-dual electromagnetic fields is also of direct physical interest: it is a solution of the coupled Einstein-Maxwell-Dirac equations, with the condensate now serving as the cosmological constant. The field equations are

$$
\begin{gathered}
\mathcal{R}_{\mu \nu}-\frac{1}{2} g_{\mu \nu} \mathcal{R}=\kappa\left(T_{\mu \nu}(\text { e.m. })+T_{\mu \nu}(\text { Dirac })\right)+\Lambda g_{\mu \nu} \\
F_{; \mu}^{\mu \nu}=0 \\
\tilde{F}_{; \mu}^{\mu \nu}=0
\end{gathered}
$$

together with the Dirac equation (11). In (11)-(5), $\omega_{\mu}^{b c}$ and $A_{\mu}$ are the spin and $U(1)$ connections. The $\gamma^{a}$ are the flat-space $\gamma$-matrices; thus $\gamma^{\mu} e_{\mu}^{a}=\gamma^{a}$ with $E_{\mu}^{a} e_{a}^{\nu}=$ $\delta_{\mu}^{\nu}, e_{\mu}^{a} \delta_{a b} e_{\nu}^{b}=g_{\mu \nu}$, etc. The Weyl spinor $\psi$ is of the form $\psi^{T}=\left(\psi_{1}, \psi_{2}, 0,0\right)$ and the matrices 
$\gamma_{a}=\tau_{1} \otimes \sigma_{a}(a=1,2,3), \gamma_{4}=\tau_{1} \otimes \mathbf{1}$ are block off-diagonal, while $\gamma_{5}=\tau_{3} \otimes \mathbf{1}$. Since the metric is Euclidean, $\psi^{\dagger}$ replaces $\bar{\psi}$ in all spinor bilinears. These lead to the automatic vanishing of spinor currents $\psi^{\dagger} \gamma_{\mu} \psi$ or $\psi^{\dagger} \gamma_{\mu} \gamma_{5} \psi$ which would otherwise have appeared on the RHS of (田) and (间).

The cosmological constant is needed since non-singular and square-integrable solutions of the SWME with $\mathcal{R}(x) \geq 0$ are forbidden by the Weitzenbock formula and Witten's vanishing theorems, which are essentially its integrated version (for a review of the SWME, see [2]). As $T_{\mu}^{\mu}($ e.m. $)=T_{\mu}^{\mu}($ Dirac $)=0$, we are clearly limited to manifolds $\mathcal{M}_{4}$ where $4 \Lambda=-\mathcal{R}$ is a positive constant, which will turn out to be provided by the monopole condensate.

It is sensible to begin with a euclideanized Bertotti-Robinson [3], 四 type Ansatz, as that solution also involves covariantly constant electromagnetic fields and admits a cosmological constant [3]. Thus, as in [3], we may try $\mathcal{M}_{4}=$ $\mathcal{M}_{2}^{(1)} \times \mathcal{M}_{2}^{(2)}$. One can then choose the conformally-flat basis one-forms

$$
e^{i}=e^{\mu} d x^{i}, \mu=\mu\left(x^{1}, x^{2}\right), i=1,2 ; \quad e^{j}=e^{\nu} d x^{j}, \nu=\nu\left(x^{3}, x^{4}\right), j=3,4
$$

and

$$
A_{\mu}=\left(A_{1}\left(x^{1}, x^{2}\right), A_{2}\left(x^{1}, x^{2}\right), A_{3}\left(x^{3}, x^{4}\right), A_{4}\left(x^{3}, x^{4}\right)\right)
$$

with no initial restriction on $\psi$. Putting the Ansatz in the SWME, however, one finds that one of $\psi_{1}$ or $\psi_{2}$ must be zero, while the other can at most be a nonvanishing constant. The cases $\psi_{1} \neq 0$ and $\psi_{2} \neq 0$ can be regarded as condensates 
of massless monopoles or antimonopoles, respectively. This is reminiscent of, and possibly dual to the gluino condensate considered by Witten [5] in the original topological twisted $N=2$ supersymmetric Yang-Mills theory.

Let us start with $\psi_{1} \neq 0$. This yields $\mathcal{R}=2 \mathcal{R}_{12}^{12}+2 \mathcal{R}_{34}^{34}=-2\left|\psi_{1}\right|^{2}$. Introducing a new constant, we put $\mathcal{R}_{12}^{12}=-|\phi|^{2}$; thus $\mathcal{R}_{34}^{34}=-\left(\left|\psi_{1}\right|^{2}-|\phi|^{2}\right)$. We now have three possibilities: (i) Both $\mathcal{M}_{2}$ 's have constant negative curvatures $\left(\left|\psi_{1}\right|>|\phi|\right)$; (ii) one $\mathcal{M}_{2}$ is flat while the other has constant negative curvature $(|\phi|=0$ or $\left.\left|\psi_{1}\right|=|\phi|\right)$; (iii) $\left|\psi_{1}\right|<|\phi|$, hence $\mathcal{M}_{2}^{(2)}=S^{2}$ and $\mathcal{M}_{2}^{(1)}$ has constant negative curvature. In all the cases, use of the Cartan structure equations, the constancy of $\left|\psi_{1}\right|$ and the SWME lead to

$$
\left(A_{1}, A_{2}\right)=-\frac{1}{2}\left(\omega_{1}^{12}, \omega_{2}^{12}\right) ; \quad\left(A_{3}, A_{4}\right)=-\frac{1}{2}\left(\omega_{3}^{34}, \omega_{4}^{34}\right)
$$

and therefore

$$
F=d A=-d \frac{1}{2}\left(\omega_{2}^{1}+\omega_{4}^{3}\right)=-\frac{1}{2}\left(R_{2}^{1}+R_{4}^{3}\right)
$$

since the curvature two-form $\mathcal{R}_{b}^{a}=\mathcal{R}_{b c d}^{a} e^{c} \wedge e^{d}$ contains no $\omega_{c}^{a} \wedge \omega_{b}^{c}$ terms in our Ansatz.

It is worth pausing briefly to consider some implications of (8) and (9). First of all, (8) and the form of $\psi$ show that the original version of Hermann Weyl's "Eichinvarianz" [6 actually holds in (11): A gauge transformation $A_{\mu} \rightarrow A_{\mu}+$ $\partial_{\mu} \alpha\left(x^{1}, x^{2}\right)+\partial_{\mu} \beta\left(x^{3}, x^{4}\right)$ can be compensated for by local changes of the scale 
factors $\mu$ and $\nu$ ! Secondly, this gauge transformation reveals that each of the two 2-manifolds effectively has its own $U(1)$ fiber. Thirdly, $\left(D_{A}\right)_{\mu} \psi=0$ by (8); hence $T_{\mu \nu}($ Dirac $)=0$ in (3). Then, contracting (3), one finds $-\mathcal{R}=2\left|\psi_{1}\right|^{2}=4 \Lambda$; hence, as mentioned before, the cosmological constant is given by the monopole condensate. Incidentally, this represents a counterexample to the folklore that solutions of the coupled Einstein-Maxwell-Dirac equations need not be sought because the Dirac field is supposed to become negligible in the classical limit where the Einstein-Maxwell equations apply.

We now seek solutions of the SWME for the cases (i)-(iii). It will be seen later that the most highly symmetric and self-dual special case of (i) also solves (355). We define the pair of complex coordinates $z^{1} \equiv x+i y \equiv \sqrt{2}|\phi|\left(x^{1}+i x^{2}\right)$, $z^{2} \equiv s+i t \equiv \sqrt{2\left(\left|\psi_{1}\right|^{2}-|\phi|^{2}\right)}\left(x^{3}+i x^{4}\right)$. The SWME then result in

$$
\begin{array}{cc}
4 \partial_{2} \partial_{\overline{2}} \nu=e^{2 \nu} \quad\left(\left|\psi_{1}\right|>|\phi|\right), \\
4 \partial_{2} \partial_{\overline{2}} \nu=0 \quad\left(\left|\psi_{1}\right|=|\phi|\right), \\
4 \partial_{2} \partial_{\overline{2}} \nu=-e^{2 \nu} \quad\left(\left|\psi_{1}\right|<|\phi|\right)
\end{array}
$$

for (i), (ii) and (iii), respectively. In addition, $\mu$ satisfies

$$
4 \partial_{1} \partial_{\overline{1}} \mu=e^{2 \mu}
$$

in all three cases. Now it is known that the Liouville equation

$$
4 \partial_{z} \partial_{\bar{z}} \Lambda= \pm e^{2 \Lambda}
$$


has the general solution [7]

$$
\Lambda=\frac{1}{2} \ln \frac{|d g / d z|^{2}}{(1 \mp g \bar{g})^{2}},
$$

where $g(z)$ is an arbitrary analytic function. Using (8), (9) and (15), the solutions of (1) and (2) now may be summarized as (we repeat (8) and (9) in order to display all the results together)

$$
\begin{gathered}
\omega^{12}=-i\left\{\frac{1}{2} d \ln \left(\frac{d \bar{g}_{1}}{d \bar{z}_{1}} \frac{d z_{1}}{d g_{1}}\right)+\frac{\left(g_{1} d \bar{g}_{1}-\bar{g}_{1} d g_{1}\right)}{\left(1-g_{1} \bar{g}_{1}\right)}\right\} \\
\omega^{34}=-i\left\{\frac{1}{2} d \ln \left(\frac{d \bar{g}_{2}}{d \bar{z}_{2}} \frac{d z_{2}}{d g_{2}}\right)+\frac{\left(g_{2} d \bar{g}_{2}-\bar{g}_{2} d g_{2}\right)}{\left(1 \mp g_{2} \bar{g}_{2}\right)}\right\}, \\
A=-\frac{1}{2}\left(\omega_{2}^{1}+\omega_{4}^{3}\right), \\
R_{2}^{1}=-2 i \frac{d g_{1} \wedge d \bar{g}_{1}}{\left(1-g_{1} \bar{g}_{1}\right)^{2}} R_{4}^{3}=-2 i \frac{d g_{2} \wedge d \bar{g}_{2}}{\left(1 \mp g_{2} \bar{g}_{2}\right)^{2}} \\
F=-\frac{1}{2}\left(R_{2}^{1}+R_{4}^{3}\right),
\end{gathered}
$$

where the upper and lower signs in (17) and (19) correspond to cases (i) and (iii), i.e., negative and positive constant curvature for $\mathcal{M}_{2}^{(2)}$. For case (ii), we may solve (11) by taking $\nu=\zeta\left(z_{2}\right)+\bar{\zeta}\left(\bar{z}_{2}\right)$, which gives $\mathcal{M}_{2}^{(2)}$ the flat metric

$$
d s^{2}=\exp \left(\zeta\left(z_{2}\right)+\bar{\zeta}\left(\bar{z}_{2}\right)\right) d z_{2} d \bar{z}_{2} \equiv d w_{2} d \bar{w}_{2}
$$




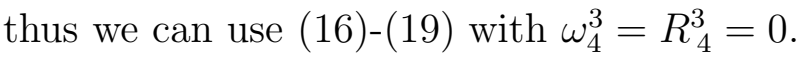

So far we have only mentioned the curvatures of the two manifolds; their global topological properties depend on the choices for $g_{1}\left(z_{1}\right), g_{2}\left(z_{2}\right)$ and $w\left(z_{2}\right)$. For example, taking $w\left(z_{2}\right)$ to be an inverse elliptic function makes $\mathcal{M}_{2}^{(2)}$ the genus $p=1$ flat torus. Similarly, but less trivially, we can tesellate the constant negative curvature hyperboloid $\left|g_{1}\left(z_{1}\right)\right| \leq 1$ by $4 p_{1}$-gons with geodesic edges. Pairwise identifying the latter in the usual way [8] turns $\mathcal{M}_{2}^{(1)}$ into a genus $p_{1}$ Riemann surface $\Sigma_{p_{1}}$. To do this explicitly, one first goes over to the Poincaré metric for the upper half-plane $C_{+}$

$$
d s^{2}=\frac{d f_{1} d \bar{f}_{1}}{\left(\operatorname{Im} f_{1}\right)^{2}}
$$

where $g_{1}\left(z_{1}\right)=\left(f_{1}-i\right) /\left(f_{1}+i\right)$, and chooses $f_{1}$ as the Fuchsian function [9], [10] used in uniformizing an algebraic function whose Riemann surface has genus $p_{1}$. Obviously, in case (i) a similar choice can be made for $g_{2}\left(z_{2}\right)$, giving $\mathcal{M}_{2}^{(2)}=\Sigma_{p_{2}}$. In case (iii), we have $\mathcal{M}_{2}^{(2)}=\Sigma_{0} \equiv S^{2}$ and the only one-to-one and onto mappings of $S^{2}$ to itself consist of $g_{2}\left(z_{2}\right)=\left(a z_{2}+b\right) /\left(c z_{2}+d\right)$.

To summarize, we have found solutions of the SWME of the form $\mathcal{M}_{4}=$ $\Sigma_{p_{1}} \times \Sigma_{p_{2}}$ where $p_{1}+p_{2} \geq 2$, excluding $p_{1}=p_{2}=1$. Using (18), the first Chern classes of the two manifolds are seen to be $p_{1}-1$ and $p_{2}-1$; these may be regarded as the number of magnetic vortices on $\mathcal{M}_{2}^{(1)}$ and electric vortices on $\mathcal{M}_{2}^{(2)}$, respectively. In the "antimonopole condensate" case with $\psi_{1}=0, \psi_{2} \neq 0$, 
the metric remains the same but $A_{\mu}$ and the first Chern classes change sign; this can be thought of as changing the sense of the vortices.

Are these solutions unique? One may first think of generating new solutions in cases (i) and (iii) via the metric-preserving transformations

$$
\tilde{g_{1}}=\frac{\alpha_{1} g_{1}+\beta_{1}}{\overline{\beta_{1}} g_{1}+\overline{\alpha_{1}}}, \tilde{g_{2}}=\frac{\alpha_{2} g_{1}+\beta_{2}}{ \pm \overline{\beta_{2}} g_{2}+\overline{\alpha_{2}}} \text {, with }\left|\alpha_{i}\right|^{2} \mp\left|\beta_{i}\right|^{2}=1 \text {, }
$$

where the upper (lower) sign again refers to (i) ((iii)). However, all the fields are seen to be invariant under such $S U(1,1) \times S U(1,1)(S U(1,1) \times S U(2))$ transformations; in particular, integer subgroups of the above only shuffle the $4-p_{i}$-gons, keeping the tesellation fixed. To investigate uniqueness more generally, one needs to compute the virtual dimension $W$ of the moduli space of the solutions via

$$
W=-(2 \chi+3 \sigma) / 4+c_{1}^{2}
$$

where the signature

$$
\sigma\left(\mathcal{M}_{4}\right)=-\frac{1}{24 \pi^{2}} \int_{\mathcal{M}_{4}} R_{b}^{a} \wedge R_{a}^{b}
$$

obviously vanishes for our solutions in which only $R_{212}^{1}$ and $R_{434}^{3}$ are non-zero. The Euler characteristic follows from the Kunneth formula

$$
\chi\left(\mathcal{M}_{4}\right)=\chi\left(\mathcal{M}_{2}^{(1)}\right) \chi\left(\mathcal{M}_{4}^{(2)}\right)=\left(2-2 p_{1}\right)\left(2-2 p_{2}\right)
$$

while, using (20), one sees that 


$$
c_{1}^{2}=\frac{1}{(2 \pi)^{2}} \int F^{2}=\chi\left(\mathcal{M}_{4}\right) / 2
$$

Hence $W=0$, proving that our SWME solutions are unique up to gauge and conformal transformations.

We return to the question of which of the above solutions also satisfy the remaining "physical" equations, of which (3) has now been reduced to

$$
\mathcal{R}_{\mu \nu}-\frac{1}{2} g_{\mu \nu} \mathcal{R}=\kappa\left(T_{\mu \nu}(\text { e.m. })\right)+\frac{1}{2}\left|\psi_{1}\right|^{2} g_{\mu \nu}
$$

Direct computation shows that only case (i) is compatible with (28) for the special value $2|\phi|=\left|\psi_{1}\right|$, which makes the curvatures of the two manifolds equal. By (8) and (9), this means the $U(1)$ field strengths are self-dual. Hence (4) and (5) are both solved and $T_{\mu \nu}($ e.m. $)=0$. The Riemannian curvature is also self-dual in the sense that the the equal sharing of the monopole condensate between the two manifolds results in their having the same curvature and the same genus; the only difference is that the vortices are "magnetic" in one and "electric" in the other. It would be interesting to investigate whether (3-5) always select the most symmetric subset of the SWME solutions.

\section{Acknowledgements}

I am grateful to S. Akbulut for patient instruction in Seiberg-Witten theory, to P. Argyres for clearing a confusing point involving (27), and to Y. Nutku for pointing out the similarities to the Bertotti-Robinson solution, carrying out the 
REDUCE check and editing. I also thank M. Arık, T. Dereli and R. Güven for helpful discussions and S. Nergiz for LATEX help.

\section{References}

[1] E. Witten, Math. Res. Lett. 1, 769 (1994).

[2] S. Akbulut, Tr. J. of Mathematics 20, 95 (1996) (Proc. of the 4th Gökova Geometry-Topology Conference).

[3] B Bertotti, Phys. Rev. 116, 1331 (1959).

[4] I Robinson, Bull. Acad. Pol. Sci. 7 , 351 (1959).

[5] E. Witten, J. Math. Phys. 35, 5101 (1994).

[6] H. Weyl, Sitzungber. Preuss. Akad. Berlin, 465 (1918).

[7] J. Liouville, J. Math. Appl. 18, 71 (1853)

[8] Dubrovin, A. T. Fomenko, and S. P. Novikov, Modern Geometry Vol. II, Springer-Verlag, NY (1985).

[9] Z. Nehari, Conformal Mapping, Dover, NY (1952).

[10] L. R. Ford, Automorphic Functions, Chelsea, NY (1951). 